\title{
Label-free Electrochemical Cocaine Aptasensor Based on a Target-inducing Aptamer Switching Conformation
}

\author{
Mei Hua,* Manlan TaO,* Ping Wang,* Yinfeng Zhang,* Zaisheng Wu,** Yanbing Chang,* and \\ Yunhui YANG ${ }^{* \dagger}$ \\ *College of Chemistry and Chemical Engineering, Yunnan Normal University, Kunming 650092, P. R. China \\ **State Key Laboratory of Chemo/Biosensing and Chemometrics, Hunan University, Changsha 410082, \\ P. R. China
}

\begin{abstract}
A novel label-free electrochemical nucleic acid aptasensor for the determination of cocaine by the immobilization of thiolated self-assembled DNA sequences on a gold nanoparticles-modified electrode is presented. When cocaine was complexed specifically to the aptamer, the configuration of the nucleic acid aptamer switched to a locked structure and the interface of the biosensor changed, resulting in a variation of the corresponding peak current of an electrochemical probe $\left(\left[\mathrm{Fe}(\mathrm{CN})_{6}\right]^{3-14-}\right)$. Cyclic voltammetry $(\mathrm{CV})$ and electrochemical impedance spectroscopy (EIS) were employed to characterize modifications of the electrode surface. The peak current was detected by differential pulse voltammetry (DPV). Under the optimized experimental conditions, the presented sensor exhibits a nice specificity towards cocaine. The decrease of the peak current response of the aptasensor has a linear relationship with the concentration of cocaine ranging from $1.0 \times 10^{-6}$ to $1.5 \times 10^{-4} \mathrm{~mol} \mathrm{~L}^{-1}$ with a detection limit of $3 \times 10^{-7} \mathrm{~mol} \mathrm{~L}^{-1}$ at $3 \sigma$. The proposed aptasensor can be easily regenerated by the denaturalization of aptamer-target complexes in a heated water bath at $80-90^{\circ} \mathrm{C}$. Besides, this biosensor has a high reproducibility and selectivity, which can be a promising method to detect cocaine in real samples.
\end{abstract}

(Received July 15, 2010; Accepted September 25, 2010; Published December 10, 2010)

\section{Introduction}

Cocaine, whose scientific name is benzoyl ecgonine, is a kind of alkaloid detached from coca leaves. ${ }^{1}$ It is well known that cocaine is one of the most serious drug abuses in the whole world. Cocaine can produce a strong psychological dependence. Long-term abuse can lead to mental disorders, also known as cocaine psychosis, which can easily result in emotional instability, self-mutilation, violence and aggressive behavior. ${ }^{2,3}$ Therefore, efficient and sensitive detection methods for cocaine are highly desired.

Various methods, such as high-performance capillary electrophoresis (HPCE), ${ }^{4}$ enzyme-linked immunosorbent assay (ELISA) ${ }^{5}$ high-performance liquid chromatography, ${ }^{6}$ gas chromatography-mass spectrometry ${ }^{7}$ and high-performance thin-layer chromatography ${ }^{8}$ have been reported for the detection of cocaine in human matrices. Although these conventional strategies provide an accurate, sensitive detection of cocaine, there are still some inconveniences, such as complicated manipulation, together with valuable apparatus that is hard-to-use in field-tests. A method with a simple procedure for the detection of cocaine is still in need of exploration.

Aptamers are one kind of single-stranded DNA or RNA sequences that could be in vitro synthesized with the systematic evolution of ligands by exponential enrichment (SELEX), ${ }^{9,10}$ which can specifically combine with target analytes due to

† To whom correspondence should be addressed.

E-mail: yyhui2002@yahoo.com.cn similar affinities, like antibody-antigen. Recently, multifarious methods have been used to improve the sensitivity and practicability in detecting cocaine. A fast colorimetric cocaine aptamer sensor has been developed by Liu and Lu. ${ }^{11}$ Also, $\mathrm{Li}$ et al. constructed an electrogenerated chemiluminescence aptamer sensor for detecting cocaine. ${ }^{12}$ Folding fluorescent sensor for cocaine has been designed by Stojanovic. ${ }^{13}$ Besides, an electrochemical aptasensor was constructed based on the high specificity and affinity of the aptamer after combining with target analytes and a change of the electrochemical signal, ${ }^{14}$ displaying many advantages, such as relatively high sensitivity, together with its simpleness, controllability, reproducibility and effectiveness. It can be easily miniaturized, which is necessary for a high-throughput system and on-site applications. Aptamer as recognition elements can be self-assembled on an electrode to fabricate an aptasensor. ${ }^{15}$ Label-free electrochemical aptasensor based on electron-transfer resistances has been proved to be an effective method to determine guest molecules (targets). ${ }^{16}$ White ${ }^{17}$ and Ceretti ${ }^{18}$ have, respectively, reported an electrochemical aptasensor based on a conformational change of the aptamer induced by a target analyte to determine cocaine and adenosine. Both of them adopt a redox label, which is complicated compared to a label-free process.

In this paper, a label-free electrochemical aptasensor based on target-inducing conformational switching of the aptamer has been fabricated. Thiolated cocaine aptamers are self-assembled onto the surface of an electrode modified with gold nanoparticles (Au NPs) by adsorption on the amine groups of chitosan. ${ }^{19} \mathrm{~A}$ relatively high current response of an electrochemical probe $\left(\left[\mathrm{Fe}(\mathrm{CN})_{6}\right]^{3-14}\right)$ can be obtained without any target analytes. 
After introducing cocaine, nucleic acid aptamers combine with cocaine to form three-way junction complexes, ${ }^{20}$ which increase the charge density and the steric hindrance on the surface of the electrode, and block the diffusion of $\left[\mathrm{Fe}(\mathrm{CN})_{6}\right]^{3-14}$ onto the electrode surface. ${ }^{21,22}$ All changes lead to an obvious decrease of the peak current of $\left[\mathrm{Fe}(\mathrm{CN})_{6}\right]^{3-/ 4}$, which is in direct proportion to a quantity of an added cocaine. This novel approach for detecting cocaine is simple and effective. It will thus be a potential application in the determination of cocaine in real samples.

\section{Experimental}

Reagents and chemicals

A cocaine aptamer ${ }^{23}\left(5^{\prime}\right.$-GGG AGA CAA GGA TAA ATC CTT CAA TGA AGT GGG TCT CCC (CH2) $\left.)_{3}-\mathrm{SH}-3^{\prime}\right)$ was synthesized by Takara Biotechnology (Dalian, China). Cocaine was purchased from the Material Evidence Identification Center of Ministry of Public Security (Beijing, China). 2-Mercaptoethanol (MCE, $\mathrm{HSCH}_{2} \mathrm{CH}_{2} \mathrm{OH}$ ) was obtained from Lanji Biological Ltd. Corp. (Shanghai, China). Chitosan (CHIT, $M_{\mathrm{W}} \sim 1 \times 10^{6} ; 75-80 \%$ deacetylation) was obtained from Sigma. All other chemicals used, such as chloroauric acid tetrahydrate $\left(\mathrm{HAuCl}_{4} \cdot 4 \mathrm{H}_{2} \mathrm{O}\right)$ and trisodium citrate $\left(\mathrm{Na}_{3} \mathrm{C}_{6} \mathrm{H}_{5} \mathrm{O}_{7} \cdot 2 \mathrm{H}_{2} \mathrm{O}\right)$, were of analytical-reagent grade, and doubly distilled water was used throughout. The assay solution was a $10 \mathrm{mmol} \mathrm{L}^{-1}$ phosphate buffer solution (PBS, pH 7.4) with $5 \mathrm{mmol} \mathrm{L}^{-1}\left[\mathrm{Fe}(\mathrm{CN})_{6}\right]^{3-14}$, which was prepared with $10 \mathrm{mmol} \mathrm{L}^{-1} \quad \mathrm{KH}_{2} \mathrm{PO}_{4}, \quad 10 \mathrm{mmol} \mathrm{L}{ }^{-1} \quad \mathrm{Na}_{2} \mathrm{HPO}_{4}, \quad 5 \mathrm{mmol} \mathrm{L}^{-1}$ $\mathrm{K}_{3} \mathrm{Fe}(\mathrm{CN})_{6}, \quad 5 \mathrm{mmol} \mathrm{L}^{-1} \quad \mathrm{~K}_{4} \mathrm{Fe}(\mathrm{CN})_{6}, \quad 0.1 \mathrm{~mol} \mathrm{~L}^{-1} \mathrm{KCl}$ and $0.1 \mathrm{~mol} \mathrm{~L}^{-1} \mathrm{NaCl}$. Serum specimens were obtained from an infirmary of Yunnan Normal University and stored at $4^{\circ} \mathrm{C}$.

\section{Apparatus}

Cyclic voltammetry (CV), electrochemical impedance spectroscopy (EIS), and differential pulse voltammetry (DPV) were carried out with a CHI 660C electrochemistry workstation (Shanghai CHI Apparatus Corp., China). Scanning electron microscopy (SEM) analysis was performed using an XL30ESEM-TMP microscope (Philips, Holand). A threeelectrode cell $(10 \mathrm{~mL})$ with a modified glassy carbon electrode as a working electrode, a saturated calomel electrode (SCE) as a reference electrode and a platinum foil electrode as a counter electrode was used. All potentials were measured and reported versus the SCE.

The preparation of a chitosan (CHIT) solution and colloidal Au NPs

A $0.2 \%$ (wt) CHIT solution was prepared by dissolving CHIT flakes into $0.05 \mathrm{~mol} \mathrm{~L}^{-1}$ acetic acid and stirred for $6 \mathrm{~h}$ at room temperature until complete dissolution. ${ }^{24}$

All glassware used in the following procedures was cleaned in a bath of aqua regia, rinsed thoroughly in double-distilled water and dried in air. Colloidal gold solutions were prepared by a well-established Frens method of reducing a $\mathrm{HAuCl}_{4}$ aqueous solution with citrate. In brief, a $100-\mathrm{mL}$ of $\mathrm{HAuCl}_{4}$ aqueous solution $(0.01 \%)$ was stirred and heated to $100^{\circ} \mathrm{C}$. Then, $2.0 \mathrm{~mL}$ of trisodium citrate $(1 \%)$ was added into a concentrated $\mathrm{HAuCl}_{4}$ aqueous solution and kept boiling for $17 \mathrm{~min}$ in order to obtain burgundy solutions. ${ }^{25}$ After cooling down, the synthesized gold colloids were stored at $4^{\circ} \mathrm{C}$ in a dark bottle.

The preparation of aptamer/Au NPs/CHIT/GCE

A glassy carbon electrode (GCE, $3 \mathrm{~mm}$ diameter) was first

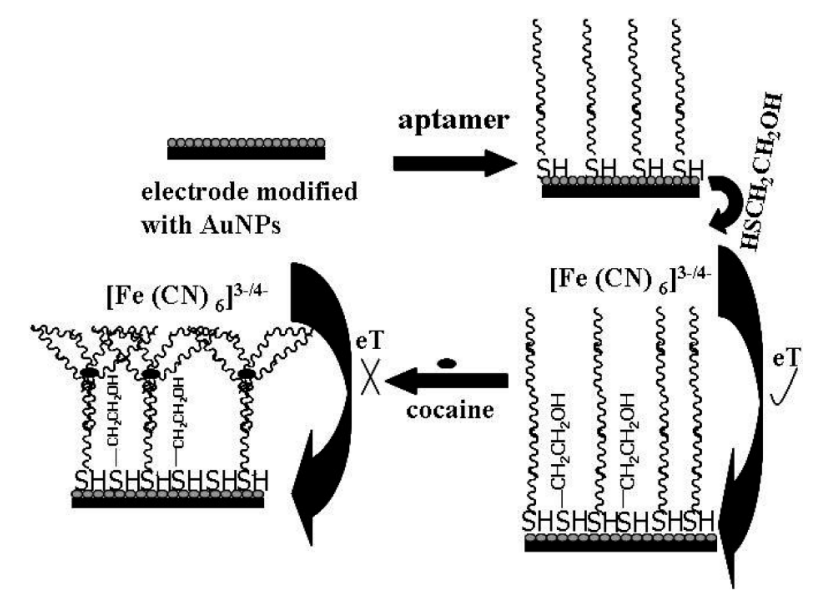

Scheme 1 Stepwise procedure of the aptasensor.

polished with emery paper and alumina slurry, successively rinsed thoroughly with absolute alcohol and distilled water in an ultrasonic bath, and dried in air. Then, $10 \mu \mathrm{L}$ of a CHIT solution was pipetted to the surface of GCE, and remained over night. A CHIT-modified electrode was immersed in a colloidal gold solution for about $12 \mathrm{~h}$ in order to adsorb Au NPs. Then, the obtained Au NPs-modified electrode was incubated in a solution of $2 \mu \mathrm{mol} \mathrm{L}-1$ cocaine aptamer for about $1 \mathrm{~h}$ at room temperature. After washing with doubly distilled water, $10 \mu \mathrm{L}$ MCE was added on the surface to any block non-specificity site. A cocaine aptasensor was obtained and kept in PBS in $4^{\circ} \mathrm{C}$ when not in use.

\section{Experimental measurements}

The electrochemical measurement was based on changes in the electrical signal after conformational switching of aptamers induced by a target molecule. ${ }^{26}$ Before a measurement, the aptasensor was incubated with various concentrations of cocaine at $37^{\circ} \mathrm{C}$. Then, the aptasensor was thoroughly rinsed with PBS $\left(10 \mathrm{mmol} \mathrm{L}^{-1} \mathrm{KH}_{2} \mathrm{PO}_{4}, 10 \mathrm{mmol} \mathrm{L}^{-1} \mathrm{Na}_{2} \mathrm{HPO}_{4}\right.$ and $\left.0.1 \mathrm{~mol} \mathrm{~L}^{-1} \mathrm{NaCl}, \mathrm{pH} 7.4\right)$. The stepwise procedure of the aptasensor combined with cocaine is shown in Scheme 1.

The electrochemical properties of the modified electrode were characterized by cyclic voltammetry and electrochemical impedances spectroscopy. Cyclic voltammetric measurements were carried out in $10 \mathrm{~mL} 0.01 \mathrm{~mol} \mathrm{~L}^{-1}$ PBS containing $5 \mathrm{mmol} \mathrm{L}^{-1}\left[\mathrm{Fe}(\mathrm{CN})_{6}\right]^{3-14-}$ at room temperature from -600 to $+800 \mathrm{mV}$ (versus $\mathrm{SCE}$ ); the scan rate was $100 \mathrm{mV} / \mathrm{s}$. Electrochemical impedances of the electrodes were performed in a background solution of $5.0 \mathrm{mmol} \mathrm{L}^{-1} \mathrm{~K}_{3} \mathrm{Fe}(\mathrm{CN})_{6}$ and $\mathrm{K}_{4} \mathrm{Fe}(\mathrm{CN})_{6}$ containing $0.1 \mathrm{~mol} \mathrm{~L}^{-1} \mathrm{KCl}$ at room temperature; the frequency range was at $10 \mathrm{mHz}$ to $10 \mathrm{kHz}$ at $220 \mathrm{mV}$ versus SCE. The differential pulse voltammetry (DPV) recorded detailed peak current was from -200 to $600 \mathrm{mV}$ (versus SCE). ${ }^{27}$

\section{Regeneration of the aptasensor}

Regeneration of the aptasensors was preformed by immersing the modified electrodes previously in hot distilled water $\left(80-90^{\circ} \mathrm{C}\right)$ for $10 \mathrm{~min}$. The regenerated transducer interface of the aptasensor was finally rinsed with distilled water and gradually cooled to room temperature before incubated with target. $^{28}$ 


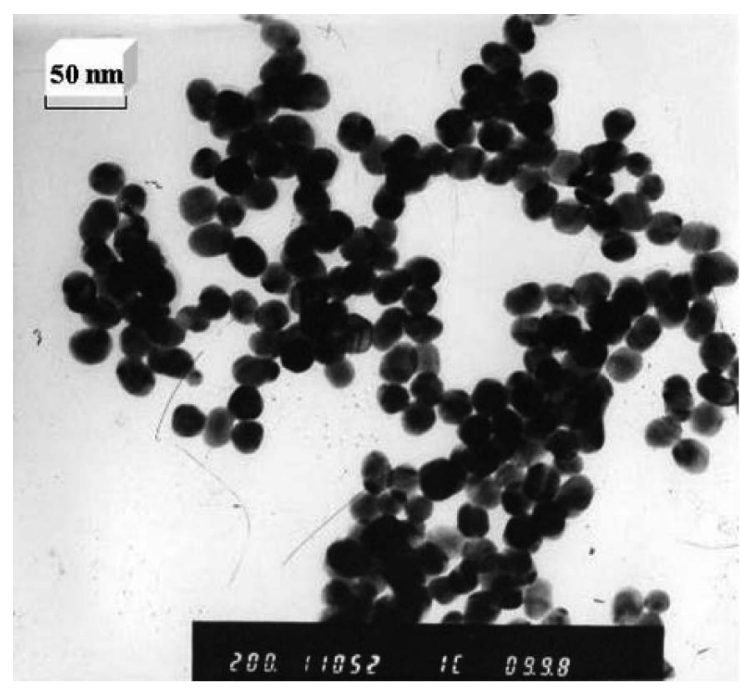

Fig. 1 TEM images of the synthesized Au NPs.

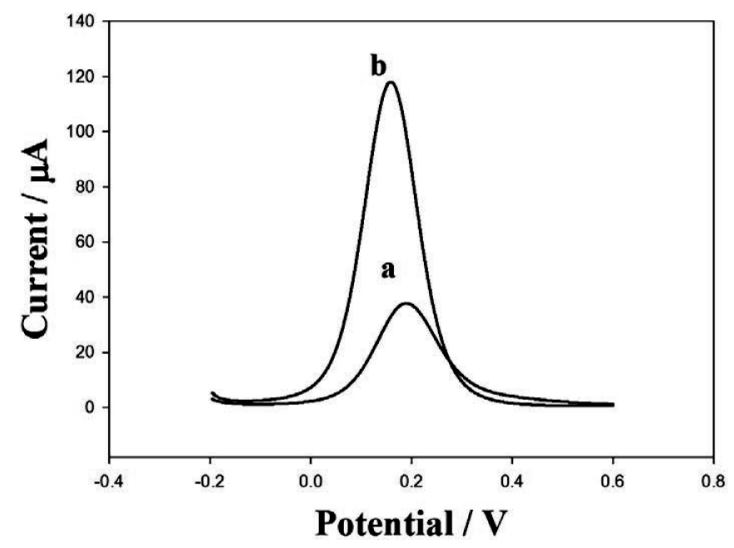

Fig. 2 Comparison of the DPV response of the flat gold electrode (a) and the electrode modified with a Au NPs (b) in $10 \mathrm{mmol} \mathrm{L}^{-1}$ phosphate buffer solution ( $\mathrm{PBS} \mathrm{pH} \mathrm{7.4)} \mathrm{with} 5 \mathrm{mmol} \mathrm{L}^{-1}\left[\mathrm{Fe}(\mathrm{CN})_{6}\right]^{3-14}$.

\section{Results and Discussion}

\section{Morphological characterization of $A u N P s$}

Figure 1 shows a transmission electron microscopy (TEM) image of Au NPs. The size of the prepared Au NPs is approximately $10 \mathrm{~nm}$, which can enlarge the specific surface area of the transducer and combine with more thiolated aptamer under the same condition.

Comparison of the performance of a flat gold electrode and the electrode modified with $\mathrm{Au} N \mathrm{NS}$

To discern the role of Au NPs, the performance of a flat gold electrode and an electrode modified with Au NPs was investigated. Figure 2 shows the DPV response of different electrodes. The peak current of the electrode modified with Au NPs (Fig. 2(b)) was higher than that of the flat gold electrode (Fig. 2(a)), which verifies the signal enhancement and advancement of the performance after a modification of $\mathrm{Au}$ NPs due to increasing the surface area of the electrode.

Cyclic voltammetric behavior of the aptasensor

Cyclic voltammetry was carried out to interrogate the

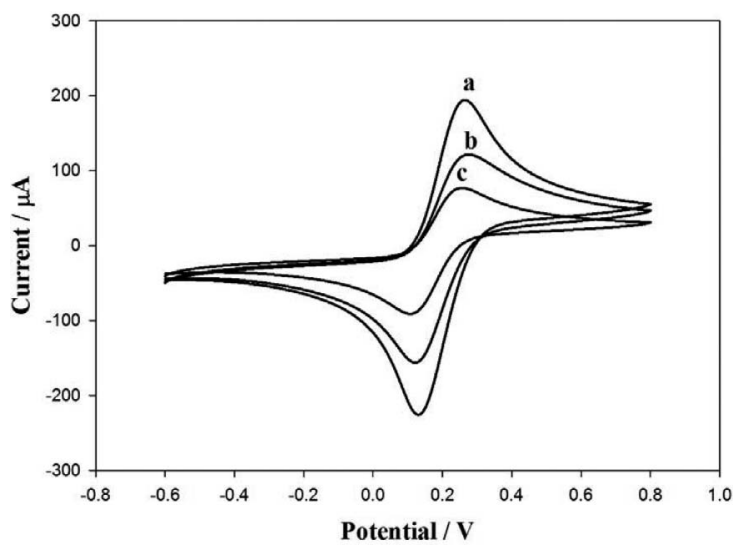

Fig. $3 \mathrm{CVs}$ of different modified electrodes in a $10 \mathrm{mmol} \mathrm{L}^{-1}$ phosphate buffer solution (PBS pH 7.4) with $5 \mathrm{mmol} \mathrm{L}^{-1}\left[\mathrm{Fe}(\mathrm{CN})_{6}\right]^{3-14}$. a, Au NPs/CHIT/GCE; b, aptamer/Au NPs/CHIT/GCE; c, aptamer/Au NPs/CHIT/GCE reacted with $20 \mu \mathrm{mol} \mathrm{L}^{-1}$ cocaine.

performance of different packing electrode. The electrode modified with Au NPs and the cocaine aptamer in the presence and absence of $20 \mu \mathrm{mol} \mathrm{L}{ }^{-1}$ cocaine were studied, respectively (Fig. 3). After the electrode was modified with the aptamer, the current response decreased compared with that of Au NPs/GCE (Fig. 3(a)), due to an electrostatic repulsion between negatively charged backbones of the aptamer and the electroactive probe (Fig. 3(b)). In the presence of cocaine, the current obviously declined, attributed to the form of three-way junctions between the aptamers and cocaine (Fig. 3(c)). A reasonable explanation is that the configurations of nucleic acid aptamers shifted when the aptamers reacted with cocaine, which hindered the diffusion of $\left[\mathrm{Fe}(\mathrm{CN})_{6}\right]^{3-14}$. Meanwhile, the scan-rate influence on the current response of the aptasensor was investigated; the result shows a typical diffusion-controlled electrochemical behavior (Fig. S1 (Supporting Information)).

A control experiment of the Au NPs/chitosan/GCE without an aptamer was performed. The current-response ratio with and without cocaine was $92.79 \%$; no obvious current variation was found, which confirms the specific effect of the analyte and the aptamer.

\section{Impedance characterization of different electrodes}

Electrochemical impedance spectroscopy (EIS) has been proven to be one of the most powerful tools for probing the features of surface-modified electrodes. ${ }^{29-31}$ Figure 4 shows the electron-transfer resistance changes $\left(\Delta R_{\mathrm{et}}\right)$ of Au NPs/CHIT/GCE (a), aptamer/Au NPs/CHIT/GCE (b) and aptamer/Au NPs/ CHIT/GCE incubated with $20 \mu \mathrm{mol} \mathrm{L}^{-1}$ cocaine (c) in $5.0 \mathrm{mmol} \mathrm{L}^{-1}\left[\mathrm{Fe}(\mathrm{CN})_{6}\right]^{3-14-}$ containing $0.1 \mathrm{~mol} \mathrm{~L}^{-1} \mathrm{KCl}$ at room temperature. It can be seen that Au NPs/CHIT/GCE exhibited a relatively low impedance value (Fig. 4(a)), which was characteristic of a diffusion-limiting step (the straight line) of the electrochemical process. An obvious change of the electrochemical impedance occurred after Au NPs/CHIT/GCE was modified with the aptamer (Fig. 4(b)), which indicated that thiolated aptamer had been immobilized on the electrode and obstructed electron-transfer. After the aptasensor reacted with cocaine, the interfacial resistance value increased again (Fig. 4(c)). It has proved that the aptamers have combined with cocaine, and then the switched structure could hinder the electron-transfer. 


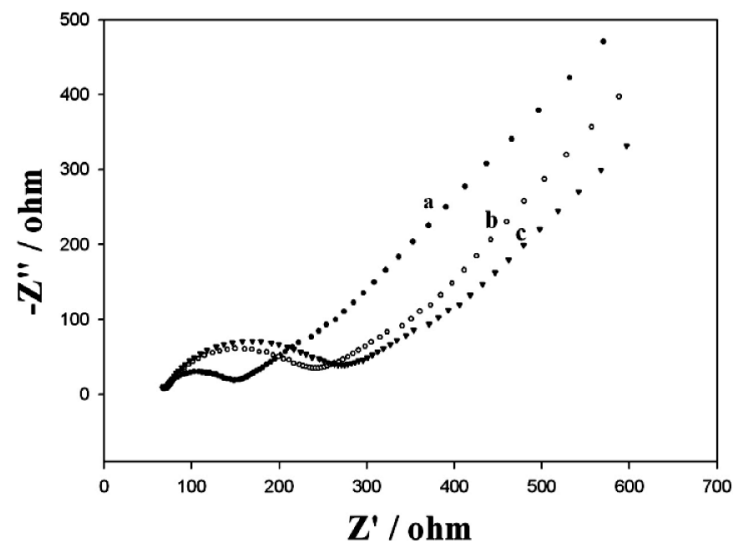

Fig. 4 Impedance spectra (Zim vs. Zre, Nyquist plot) of different modified electrodes in a $10 \mathrm{mmol} \mathrm{L}^{-1}$ phosphate buffer solution (PBS pH 7.4) with $5 \mathrm{mmol} \mathrm{L}^{-1}\left[\mathrm{Fe}(\mathrm{CN})_{6}\right]^{3-/ 4}$. a, Au NPs/CHIT/GCE; b, aptamer/Au NPs/CHIT/GCE; c, aptamer/Au NPs/CHIT/GCE reacted with $20 \mu \mathrm{mol} \mathrm{L}^{-1}$ cocaine.

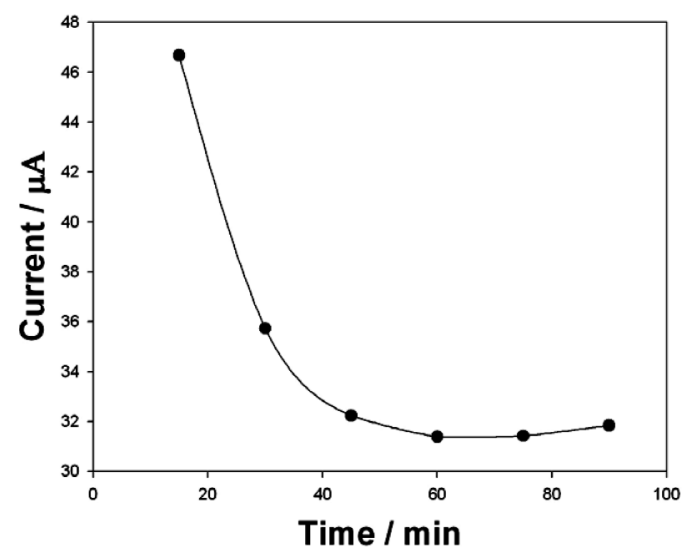

Fig. 5 Effect of the incubation time of cocaine.

\section{Effect of the incubation time of cocaine}

Different incubation times of cocaine might cause an obvious difference in the response peak current, which presents the optimal reaction time between the aptamer and its target. Thus, a series of times were investigated to find theoptimum incubation time using the same analyte concentrations in the study (Fig. 5). It took some time to form steady complexes after the target analytes were incubated on the surface of the aptasensor. The results indicate that the current responses decreased rapidly with the incubation time, and then slowed down, and reached a plateau. After that, the combination was saturated because the inherent association constant of the aptamer-target. Considering that the present aptasensor belongs to signal off, a incubation time of 60 min was adopted in subsequent experiments. The $\mathrm{pH}$ of an electrolyte solution may also affect the optimal current response; the results are listed in Fig. S2 (Supporting Information).

The response characteristics of aptamer/Au NPs/CHIT/GCE

Figure 6 shows the DPV response of aptamer/Au NPs/ CHIT/GCE for different cocaine concentrations (A), and a calibration curve for cocaine detection with the proposed aptasensor under the optimal experimental conditions (B). As expected, the DPV peak current decreased with an increase in
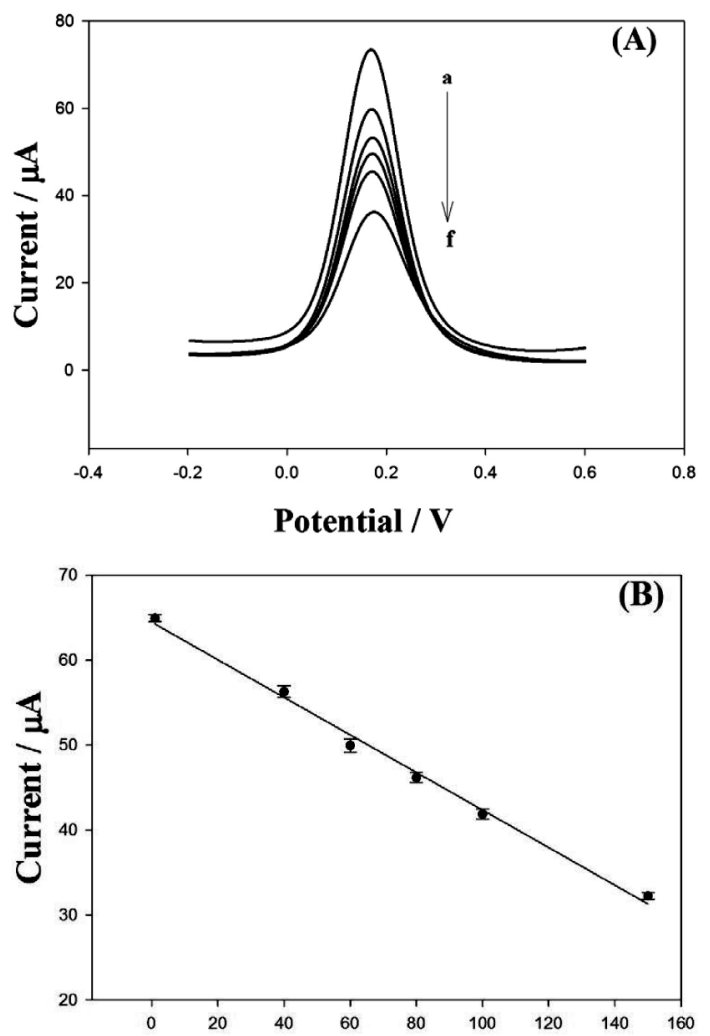

The concentration of cocaine / $\boldsymbol{u M}$

Fig. 6 Response characteristics of the aptasensor. (A) DPV response of the aptasensor for different cocaine concentrations. (B) Calibration curves of the aptasensor.

the cocaine concentration (signal off). The linear range covered from $1 \times 10^{-6}$ to $1.5 \times 10^{-4} \mathrm{~mol} \mathrm{~L}^{-1}$ with a regression equation of $I\left(10^{-6} \mathrm{~A}\right)=-0.221 C(\mu \mathrm{mol} \mathrm{L}-1)+64.46$ and a correlation coefficient of 0.9938 . The detection limit of the sensor was $3 \times 10^{-7} \mathrm{~mol} \mathrm{~L}^{-1}$ at $3 \sigma$, which is superior to the value of $1 \times 10^{-5} \mathrm{~mol} \mathrm{~L}^{-1}$ reported by Golub, using an aptasensor labeled with Pt nanoparticles to detect cocaine..$^{32}$

\section{Specificity of an aptasensor}

Some probable interfering substances have been chosen to explore the specificity of an aptasensor. An aptasensor without any targets produced a relative high peak current (Fig. 7(a)) compared with that of an aptasensor incubated with $100 \mu \mathrm{mol} \mathrm{L}-1$ cocaine (Fig. 7(h)). The reason is that the conformational switching of the aptamer leads to a decline in the peak current after combining with cocaine. The curves from $b$ to $g$ were obtained after an aptasensor was incubated with $4 \mathrm{mmol} \mathrm{L}^{-1}$ nicotine, ascorbic acid, caffeine, glucose, morphine, and uric acid, respectively. It can be seen that interferents with 30 -fold to the cocaine concentration only caused a slight decline in the peak current after reacting with the aptasensor; it can thus be concluded that those substances can hardly specifically combine with the aptasensor, illustrating the relative high selectivity of the aptasensor.

\section{The repeatability of aptasensor}

After performing 200-cyclic voltammetry in the same supporting electrolyte, the responses of current was found to be $88.93 \%$ of the original, which demonstrates the good repeatability of the aptasensor. 


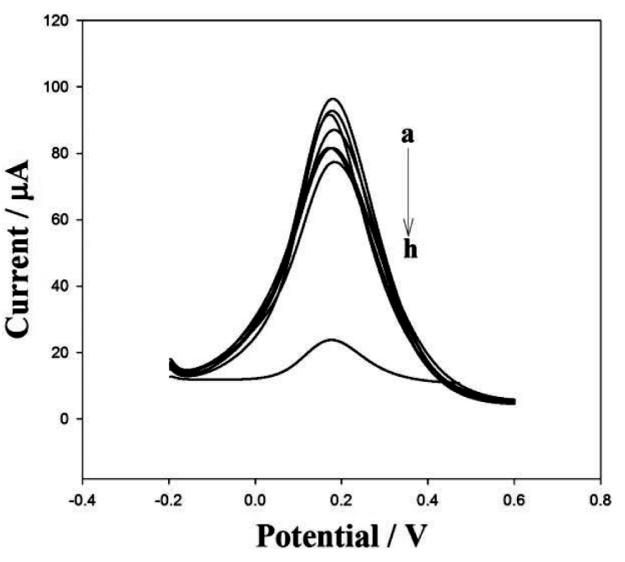

Fig. 7 Specificity of the aptasensor. DPV response of the aptasensor without any targets (a); incubated with $4 \mathrm{mmol} \mathrm{L}^{-1}$ nicotine, ascorbic acid, caffeine, glucose, morphine, uric acid, respectively (b to g) and incubated with $100 \mu \mathrm{mol} \mathrm{L}^{-1}$ cocaine (h).

Table 1 Recovery of the proposed biosensor

\begin{tabular}{cccc}
\hline Sample & Added $/ \mu \mathrm{mol} \mathrm{L}{ }^{-1}$ & Found $/ \mu \mathrm{mol} \mathrm{L}^{-1}$ & Recovery, \% \\
\hline 1 & 50.0 & 45.2 & 90.4 \\
2 & 70.0 & 68.1 & 97.3 \\
3 & 90.0 & 91.3 & 101.4 \\
\hline
\end{tabular}

The regenerated aptasensor has been used to identify the activity of the sensor surface. In brief, the successive regenerated aptasensor had incubated with $60 \mu \mathrm{mol} \mathrm{L}{ }^{-1}$ cocaine more than 5 times; the RSD was $2.9 \%$, which is better than our previous work using a nano-gold and CHIT hybrid film electrochemical co-deposited on a GCE. ${ }^{33}$ That is to say, while using the denaturalization processes, there no gold nanoparticles apparently leaked. According to our previous research, Au NPs can be adsorbed on the amine groups of some compounds through $\mathrm{Au}-\mathrm{NH}_{2}$ bond, which is very stably and can only be destroyed via a saline solution with acid and alkali successively. ${ }^{34}$ This characteristic ensures the following combination of cocaine, and further improves the repeatability of the aptasensor.

\section{Determination of cocaine in blood serum}

In order to confirm the practicability of the aptasensor, the standard addition method was used to detect cocaine in real diluted serum samples. To investigate the effect of plasma on the response of the aptasensor, the constructed aptasensor was first used to detect the same concentration of cocaine with and without the addition of a plasma matrix. The current response ratio with and without a plasma was $98.19 \%$, which indicates that there is only a very small unspecific effect of the plasma on the current response. Thus, a $1.0-\mathrm{mL}$ serum sample was added into $10 \mathrm{~mL}$ electrolyte containing $5 \mathrm{mmol} \mathrm{L}^{-1} \mathrm{~K}_{3} \mathrm{Fe}(\mathrm{CN})_{6}$, 5 mmol L-1 $\mathrm{K}_{4} \mathrm{Fe}(\mathrm{CN})_{6}$ and $0.1 \mathrm{~mol} \mathrm{~L}^{-1} \mathrm{KCl}$ to obtain a diluted serum sample. The recovery is elaborated in Table 1.

\section{Conclusions}

In this paper, the change in the mass transfer resistance of a reporter molecule, $\left[\mathrm{Fe}(\mathrm{CN})_{6}\right]^{3-14-}$, to the electrode surface to monitor the change in the conformation of a DNA aptamer in the presence of cocaine was used to construct a label-free aptamer-based sensor. When cocaine was present, the aptamer self-assembled on gold nanoparticles-modified electrode folds to bind the cocaine, and as such created a change in the charge structure and sterics at the electrode surface, which in turn changed (increased) the mass-transfer resistance of the electrochemical probe, together with the change in the electrical signal. The decline in the peak current showed good linearity with the concentration of cocaine. This proposed electrochemical aptasensor for detecting cocaine is label-free, controllable, reproducible, effective and easy to prepare, together with high selectivity, which can be extended to being applied in clinic diagnostics and forensic analysis.

\section{Acknowledgements}

This work was supported by the National Natural Science Foundation of China (Grants No. 20865006), the Foundation of Science Commission of Yunnan Province (No. 2006B0028M) and the Foundation of Department of Education of Yunnan Province (No. 07Z10087).

\section{Supporting Information}

Scan rate influence on the current response of the aptasensor and effect of $\mathrm{pH}$ on the response of aptasensor. This material is available free of charge on the Web at http://www.jsac.or.jp/ analsci/.

\section{References}

1. R. Xia and H. X. Ma, Modern Preventive Medicine, 2007, 34, 2901.

2. L. Fattore, M. C. Puddu, and S. Picciau, Neuroscience, 2002, 110,1

3. L. D. Kramer, G. E. Locke, and A. Ogunyemi, Am. J. Drug Alcohol Abuse, 1990, 16, 307.

4. Q. Y. Jin, C. Liang, and Y. M. Guo, Chin. J. Clin. Pharm., 2003, 12, 285.

5. M. L. Pujol, V. Cirimele, and P. J. Tritsch, Forensic Sci. Int., 2007, 170, 189.

6. L. Virag, B. Mets, and S. Jamdar, J. Chromatogr., B, 1996, 681, 263.

7. I. Alvarez, F. Palos, and A. M. Bermejo, Anal. Lett., 2006, 39, 1393.

8. M. Yonamine and M. C. Sampaio, Legal Med., 2006, 8, 184.

9. A. D. Ellington and J. W. Szostak, Nature, 1990, 346, 818.

10. K. Ohsawa, T. Kasamatsu, J. Nagashima, K. Hanawa, M. Kuwahara, H. Ozaki, and H. Sawai, Anal. Sci., 2008, 24 , 167.

11. J. W. Liu and Y. Lu, Angew. Chem., Int. Ed., 2006, 45, 90.

12. Y. Li, H. L. Qi, Y. G. Peng, J. Yang, and C. X. Zhang, Electrochem. Commun., 2007, 9, 2571.

13. M. N. Stojanovic, P. D. Prada, and D. W. Landry, J. Am. Chem. Soc., 2001, 123, 4928.

14. K. Ikebukuro, C. Kiyohara, and K. Sode, Biosens. Bioelectron., 2005, 20, 2168.

15. L. H. Lei, Y. C. Fu, X. H. Xu, Q. J. Xie, and S. Z. Yao, Prog. Chem., 2009, 21, 724.

16. M. Macini, "Aptamers in Bioanalysis", 2009, John Wiley and Sons, Hoboken, NJ.

17. R. J. White and K. W. Plaxc, Proc. SPIE., 2009, 21, 
732105-1.

18. H. Ceretti, B. Ponce, S. A. Ramírez, and J. M. Montserrat, Electroanalysis, 2010, 22, 147.

19. Y. L. Zhou, J. F. Zhi, J. W. Zhao, and M. T. Xu, Anal. Sci., 2010, 26, 957.

20. M. F. Huang, Y. C. Kuo, and C. C. Huang, Anal. Chem., 2004, 76, 192.

21. Y. Lu, X. Li, and L. Zhang, Anal. Chem., 2008, 80, 1883.

22. D. K. Xu, D. W. Xu, and X. B. Yu, Anal. Chem., 2005, 77, 5107.

23. J. W. Liu, D. Mazumdar, and Y. Lu, Angew. Chem., Int. Ed., 2006, 45, 7955.

24. L. Tan, G. M. Yang, Z. Y. Xie, H. P. Bai, X. X. Lu, and Y. H. Yang, Anal. Lett., 2008, 41, 2860.

25. R. Q. Feng, Z. Q. Guo, and J. B. Mi, "Modern Technology and Application of Antibody", ed. Peking University, 2006, China.

26. M. N. Stojanovic and D. W. Landry, J. Am. Chem. Soc.,
2002, 124, 9678.

27. Z. S. Wu, M. M. Guo, S. B. Zhang, C. R. Chen, J. H. Jiang, and G. L. Shen, Anal. Chem., 2007, 79, 2933.

28. F. Zheng, Z. S. Wu, S. B. Zhang, M. M. Guo, C. R. Chen, G. L. Shen, and R. Q. Yu, Chem. Res. Chin. Univ., 2008, 24, 138.

29. Z. L. Cheng, E. K. Wang, and X. R. Yang, Biosens. Bioelectron., 2007, 22, 1716.

30. Z. M. Liu, Z. J. Li, G. L. Shen, and R. Q. Yu, Electroanalysis, 2009, 21, s1781.

31. X. X. Li, Life Sci. Instruments, 2009, 7, 27.

32. E. Golub, G. Pelossof, R. Freeman, H. Zhang, and I. Willner, Anal. Chem., 2009, 81, 9291.

33. G. M. Yang, Y. B. Chang, H. Yang, L. Tan, Z. S. Wu, X. X. Lu, and Y. H. Yang, Anal. Chim. Acta, 2009, 644, 72.

34. Y. H. Yang, Z. J. Wang, M. M. Guo, M. H. Yang, Z. Y. Wu, G. L. Shen, and R. Q. Yu, Sens. Actuators, B, 2005, 114, 1. 